Recepción: 13 / 02 / 2018

Aceptación: 09 / 04 / 2018

Publicación: 05 / 06 / 2018

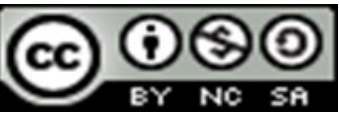

Ciencias de la Salud

Articulo Científico

\title{
Causas y manejo del dolor postoperatorio de la paciente obstétrica
}

\section{Causes and management of postoperative pain of the obstetric patient}

\section{Causas e manejo da dor pós-operatória do paciente obstétrico}

\author{
Gema M. Loor-Muñoz ${ }^{\mathrm{I}}$ \\ gemamon@yahoo.com \\ Grécia C. León-Álava II \\ grteciacecilia@gmail.com \\ María M. Menéndez-Cañarte ${ }^{\mathrm{III}}$ \\ victor@gmail.com
}

Correspondencia: gemamon@hotmail.com

\footnotetext{
I Médico Cirujano, Residente Asistencial del Área de Anestesiología del Hospital Rodríguez Zambrano, Manta, Ecuador.

${ }^{\text {II }}$ Magister en Gerencia Clínica en Salud Sexual y Reproductiva, Especialista En Anestesiología, Doctora en Medicina y Cirugía, Anestesiólogo Gineco-obstetra en Centro Obstétrico del Hospital Rodríguez Zambrano, Manta, Ecuador.

III Doctora Medicina y Cirugía, Residente Asistencial del Área de Anestesiología del Hospital Rodríguez Zambrano, Manta, Ecuador.
} 


\title{
Resumen
}

El manejo del dolor posterior al parto o a la cesárea es un procedimiento frecuente; el parto se asocia con trauma físico y casi un tercio de éstos se realizan por cesárea. La solicitud de una mujer embarazada de alivio del dolor, es suficiente motivo para su indicación médica. En este artículo se evaluó el manejo oportuno y adecuado del dolor en pacientes con puerperio quirúrgico hospitalizadas en el servicio de obstetricia del hospital General Dr. Rafael Rodríguez Zambrano del cantón de Manta. La metodología fue de tipo descriptiva hermenéutica. La población estuvo constituida por 82 pacientes de obstetricia que fueron atendidas durante el primer trimestre del 2017. Los instrumentos utilizados fue una hoja de registro de los datos sociodemográficos y obstétricos aportados por la paciente y la medición del dolor a través del instrumento que presenta Mc Caffery y Pasero. Los resultados del análisis de los datos indicaron que predomino el dolor moderado según su intensidad, mientras que el dolor máximo presentado en las primeras 24 horas fue clasificado como severo, por un $48 \%$ de las mujeres, el dolor moderado se presentó con un $37 \%$. En las conclusiones, se evidencio que no existe un manejo adecuado del dolor en pacientes puerperio atendidas por el servicio de obstetricia, reflejado en la manifestación de dolor durante las primeras 24 horas al ser clasificado como severo y con un dolor promedio moderado.

Palabras clave: dolor postoperatorio; paciente obstétrica; cesáreo y parto.

\begin{abstract}
Postpartum pain management or cesarean section is a frequent procedure; childbirth is associated with physical trauma and almost a third of these are performed by cesarean section. The request of a pregnant woman for pain relief is sufficient reason for her medical indication. In this article we evaluated the opportune and adequate management of pain in patients with postpartum surgery hospitalized in the obstetrics service of the General Dr. Rafael Rodríguez Zambrano Hospital in the canton of Manta. The methodology was descriptive hermeneutical type. The population consisted of 82 obstetric patients who were attended during the first quarter of 2017. The instruments used were a record sheet of sociodemographic and obstetric data provided by the patient and the measurement of pain through the instrument presented by Mc Caffery and Pasero. The results of the analysis of the data indicated that moderate pain predominated according to its intensity, while the maximum pain presented in the first 24 hours was classified as severe, by
\end{abstract}


$48 \%$ of women, moderate pain was presented with $37 \%$. In the conclusions, it was evidenced that there is no adequate management of pain in puerperium patients attended by the obstetrics service, reflected in the manifestation of pain during the first 24 hours to be classified as severe and with a moderate average pain.

Keywords: postoperative pain; obstetric patient; caesarean and delivery.

\section{Resumo}

O manejo da dor pós-parto ou cesárea é um procedimento frequente; o parto está associado a trauma físico e quase um terço destes são realizados por cesariana. O pedido de uma mulher grávida para alívio da dor é motivo suficiente para sua indicação médica. Neste artigo avaliamos o manejo oportuno e adequado da dor em pacientes com cirurgia no pós-parto internados no serviço de obstetrícia do Hospital Geral Dr. Rafael Rodríguez Zambrano, no cantão de Manta. A metodologia foi do tipo hermenêutico descritivo. A população era de 82 pacientes de obstetrícia que foram tratados durante o primeiro trimestre de 2017. Os instrumentos utilizados foi uma folha de registo de dados sociodemográficas e obstétricas fornecidos pela medição paciente e dor através do instrumento tendo Mc Caffery e Pasero. Os resultados da análise dos dados indicaram que a dor moderada predominou de acordo com sua intensidade, enquanto a dor máxima apresentada nas primeiras 24 horas foi classificada como severa, por $48 \%$ das mulheres, dor moderada foi apresentada com 37\%. Nas conclusões, evidenciou-se que não há manejo adequado da dor no puerpério assistido pelo serviço de obstetrícia, refletido na manifestação da dor nas primeiras 24 horas para ser classificada como severa e com dor média moderada.

Palavras chave: dor pós-operatória; paciente obstétrica; cesárea e parto.

\section{Introducción}

El dolor se define como una experiencia sensorial y emocional no placentera; asociado a un daño tisular, el cual es real o potencial, o bien descrito en términos de dicho daño. De tal forma que, en la paciente obstétrica, el dolor por causas relacionadas con el embarazo o el nacimiento es subjetivo y multifactorial, así mismo, es una experiencia única e irrepetible. Durante el embarazo hay una alta incidencia de dolor en abdomen, región lumbosacra y en la pelvis, secundario a los cambios fisiológicos, lo que podría aumentar la incidencia observada de dolor crónico postparto. 
El dolor del parto es una de las experiencias más significativas en la vida de una mujer. Un trabajo de parto doloroso produce cambios fisiológicos maternos que pueden afectar el bienestar materno y fetal. Al respecto, Covarrubias et al (2006), afirma que, en la paciente obstétrica, la percepción del dolor es el resultado de una experiencia única y multifactorial, la cual es influenciada por la ansiedad, las experiencias previas, los aspectos étnico-culturales y el medio ambiente.

La liberación de catecolaminas maternas en especial de noradrenalina y epinefrina en respuesta al dolor de parto aumenta el consumo de oxígeno de la madre, lo que puede conducir a una actividad uterina mal coordinada, provocando en consecuencia una disminución en la perfusión placentaria.

Al respecto cabe señalar que el intenso dolor del parto se ha correlacionado con el desarrollo de estrés postraumático después del parto, la depresión posparto y dolor persistente. Asimismo, la cesárea es una de las principales cirugías realizadas en los Departamentos de Obstetricia/Ginecología y su tasa de incidencia está aumentando debido a distintas causas, como aumento de la edad en contraer matrimonio y el nivel socioeconómico de la comunidad, entre otros. En el dolor por cesárea, la incisión quirúrgica es la responsable de liberación de sustancias nociceptivas que estimulan a los nociceptores periféricos, activando a las fibras relativas de tipo C y A-delta. Del mismo modo, las contracciones uterinas post-cesárea son las responsables de la activación de los mecanorreceptores, liberando de sustancias responsables de la inmunidad celular y del proceso inflamatorio. En ambos casos, ya sea por trabajo de parto o cesárea, esta liberación de sustancias, activa a los mecanismos de sensibilización periférica y central, por lo que respuestas sensitivas como alodinia e hiperalgesia secundaria pueden estar presentes.

De allí, que el manejo inadecuado del dolor postoperatorio en postparto o postcesárea puede afectar de forma significativa el bienestar de la madre y el neonato; esto es debido a que en presencia de dolor se retrasa la deambulación, el tiempo de inicio de la ingesta y la ventilación presenta un patrón restrictivo que condiciona a la acumulación de secreciones. Estas alteraciones, en conjunto, favorecen el desarrollo de complicaciones como lo son el íleo, atelectasias, neumonía, trombo embolismos; así mismo, la liberación de catecolaminas inhibe la secreción de oxitocina, con lo cual la producción de leche materna disminuye o se suspende. 


\section{Manejo del dolor durante el trabajo de parto}

El estándar de manejo es una analgesia de la columna vertebral, para Whizar (2009), significa controlar el dolor desde la cintura hacia abajo. Los tipos de anestesia que pueden indicarse durante el trabajo de parto y el parto mismo son:

a. Anestesia peridural: es el tipo más frecuente de analgesia en el trabajo de parto es la denominada peridural en la que el médico instala un catéter por fuera del saco de líquido que rodea la médula espinal, denominado espacio epidural, mediante el que inyecta un medicamento que insensibiliza o bloquea la sensibilidad para que el paciente no experimente dolor. A través de este catéter, la paciente puede recibir más medicamentos en la medida en que lo necesite sin necesidad de una nueva punción.

b. Anestesia combinada espinal-epidural: en ella se combina una anestesia espinal llamada también raquídea con una anestesia peridural para aprovechar las ventajas de ambas. Este tipo de anestesia comienza a hacer efecto inmediatamente y funciona bien para procedimientos más cortos mientras que la anestesia epidural es más indicada para procedimientos más prolongados. De allí que la combinación de ambas suele ser una buena opción para manejar el dolor.

c. Alternativas analgésicas: existen otras alternativas analgésicas. Sin embargo, poseen una menor efectividad en el alivio del dolor y pudieran tener más efectos indeseados que la llamada anestesia del neuroeje. Entre estas se incluyen los fármacos opioides o endovenosos y/o la inhalación de óxido nitroso, que tiene efecto analgésico.

d. Alternativas no farmacológicas: pueden ser utilizadas como complemento para retrasar la administración de la anestesia medicamentosa e incluyen técnicas como la hipnosis, reiki, yoga y esferoterapia, las que son realizadas en la sala de preparto, también son alternativas la terapia de calor y frío, el hipnotismo, la musicoterapia y aromaterapia. En un estudio realizado por Olapour et al (2013), para el alivio del dolor después de la cesárea, encontraron una reducción significativa en el dolor después de la inhalación de lavanda y podría ser utilizado como una parte del tratamiento analgésico multimodal después de una cesárea. 


\section{Metodología}

El estudio siguió una metodología de tipo descriptiva - transversal y con un enfoque epistémico hermenéutico. La población estuvo constituida por 82 pacientes con puerperio quirúrgico, hospitalizadas en el servicio de obstetricia del hospital General Dr. Rafael Rodríguez Zambrano del cantón de Manta, y fueron atendidas durante el primer trimestre del año 2017. Los instrumentos utilizados fue una hoja de registro que solicitaba información a las pacientes acerca de los datos sociodemográficos y obstétricos y la medición del dolor a través del instrumento tipo cuestionario que presenta Mc Caffery y Pasero (1999), el cual mide la intensidad del dolor, así como, el nivel de satisfacción con el manejo del dolor por parte de los médicos y el personal de enfermería. Es un instrumento confiable y está compuesto por 9 reactivos los cuales se miden de diferente manera, los primeros cuatro miden la presencia e intensidad del dolor por medio de la escala visual numérica con valores de 0 a 10 , donde 0 es ausencia de dolor y 10 es el máximo dolor. Los datos aportados se procesaron estadísticamente considerando el análisis descriptivo y de medias a través del paquete estadístico del SPSS versión 23.

\section{Análisis y discusión de los resultados}

A continuación, se presenta los resultados más representativos de la información obtenida a partir de la hoja de registro que solicitaba información a las pacientes acerca de los datos sociodemográficos y obstétricos y de la aplicación del instrumento tipo cuestionario que presenta Mc Caffery y Pasero (1999).

\section{Características de las mujeres participantes}

La edad promedio fue de 23 años con una desviación estándar de 4,7 y su nivel de escolaridad de 11 años con una desviación estándar de 4,2. El número de embarazos fue entre 2 y 3 , y contaba con una cesárea previa. 
Tabla 1

Análisis Descriptivo del Indicador Edad y Nivel de escolaridad

\begin{tabular}{l|c}
\hline \multicolumn{1}{c|}{$\begin{array}{c}\text { Análisis/ } \\
\text { Alternativas }\end{array}$} & Media \pm DE \\
\hline \hline Edad & $\mathbf{2 3} \pm \mathbf{4 , 7}$ \\
Nivel de Escolaridad & \\
& $\mathbf{1 1} \pm \mathbf{4 , 2}$ \\
\hline \hline
\end{tabular}

Fuente: Elaboración propia.

En cuanto al tipo de cesárea según el antecedente obstétrico predominó la cesárea anterior con $61,5 \%$, según la indicación de la cesárea fue la de urgencia con 72.4\% y según la técnica quirúrgica fue la Beck con $78 \%$.

Tabla 2

Análisis Descriptivo del Indicador de Antecedentes Obstétrico

\begin{tabular}{|c|c|c|c|c|}
\hline \multirow{3}{*}{$\begin{array}{c}\text { Análisis/ } \\
\text { Alternativas }\end{array}$} & \multicolumn{4}{|c|}{ Pacientes } \\
\hline & Sí & & & \\
\hline & $\mathbf{F r}$ & $\%$ & $\mathbf{F r}$ & $\%$ \\
\hline Cesaría anterior & 54 & $\overline{61,5}$ & 28 & 38,5 \\
\hline Cesaría de urgencia & 59 & 72.4 & 23 & 27,6 \\
\hline Técnica quirúrgica & 64 & 78 & 18 & 22 \\
\hline
\end{tabular}

Fuente: Elaboración propia.

Las mujeres del estudio refirieron recibir orientación preoperatoria en un $78.6 \%$ de las cuales el $45.6 \%$ indico que recibió la orientación del médico, anestesiólogo y enfermera y un 4.7 señalo que la recibieron del médico y del anestesiólogo. 
Tabla 3

Análisis Descriptivo del Indicador Orientación preoperatoria

\begin{tabular}{l|l|l|c|c|}
\hline \multirow{2}{*}{$\begin{array}{c}\text { Análisis/ } \\
\text { Alternativas }\end{array}$} & \multicolumn{5}{|c}{ Sacientes } \\
\cline { 2 - 5 } & Fr & $\%$ & Fr & \% \\
\hline \hline Orientación Preoperatoria & 64 & 78.6 & 18 & 21.4 \\
$\begin{array}{l}\text { Orientación del médico, } \\
\text { anestesiólogo y enfermera }\end{array}$ & 37 & 45.6 & 45 & 63 \\
$\begin{array}{l}\text { Orientación del médico y } \\
\text { anestesiólogo }\end{array}$ & 4 & 4.7 & 78 & 95.3 \\
\hline \hline
\end{tabular}

Fuente: Elaboración propia.

\section{Características del dolor según el test de Mc Caffery y Pasero (1999)}

El dolor máximo en las primeras 24 horas que refirieron las mujeres obtuvo una media de 6,8 con una desviación estándar de 1,80 y el dolor al momento de solicitarles la información fue de 5.3 con una desviación estándar de 1,4, en una escala del 1 al 10.

Tabla 4

Análisis Descriptivo del Indicador Dolor

\begin{tabular}{l|c}
\hline \multicolumn{1}{c|}{$\begin{array}{c}\text { Análisis/ } \\
\text { Alternativas }\end{array}$} & Media $\pm \mathbf{D E}$ \\
\hline \hline Dolor Máximo las primeras 24 horas & $\mathbf{6 . 8} \pm \mathbf{1 , 8}$ \\
Dolor en las primeras 24 horas & $\mathbf{5 , 3} \pm \mathbf{1 , 4}$ \\
\hline \hline
\end{tabular}

Fuente: Elaboración propia. 
Predomino el dolor moderado según su intensidad, mientras que el dolor máximo presentado en las primeras 24 horas fue clasificado como severo, por un $48 \%$ de las mujeres, el dolor moderado se presentó con un 37\%. Por otro lado, de acuerdo a la clasificación de la intensidad del dolor, las mujeres con cesárea anterior 49,8\%, cesárea electiva 46\% y cesárea tipo Beck, tuvieron un dolor moderado. Para la intensidad del dolor según las cirugías previas y las que recibieron orientación preoperatoria, fue clasificada por las mujeres del estudio como dolor moderado con 53.3\% y $51.9 \%$ respectivamente. Es importante puntualizar que un $17 \%$ de las mujeres con cirugía previa refirió un dolor intenso, de forma similar lo reportan las mujeres que no recibieron orientación preoperatoria $17.8 \%$

Tabla 5

Análisis Descriptivo del Indicador Intensidad del dolor

\begin{tabular}{l|l|l|l|c|}
\hline \hline \multirow{2}{*}{\multicolumn{1}{c}{$\begin{array}{c}\text { Análisis/ } \\
\text { Alternativas }\end{array}$}} & \multicolumn{4}{|c}{ Sacientes } \\
\cline { 2 - 5 } & \multicolumn{1}{|c|}{ Fr } & $\%$ & Fr & No \\
\hline Ausencia del dolor & 4 & 5 & 78 & 95 \\
Dolor Leve & 9 & 11 & 73 & 89 \\
Dolor Moderado & 30 & 37 & 52 & 63 \\
Dolor Severo & 39 & 48 & 43 & 52 \\
\hline \hline
\end{tabular}

Fuente: Elaboración propia.

El tiempo de espera para recibir el analgésico cuando reportaron las mujeres tener dolor, tardó menos de 15 minutos en el 72\%. El 18\% indicaron que nunca solicitaron medicamentos para el dolor.

Tabla 6

Análisis Descriptivo del Indicador Tiempo de espera

\begin{tabular}{l|l|l|l|c|}
\hline \hline \multirow{2}{*}{$\begin{array}{c}\text { Análisis/ } \\
\text { Alternativas }\end{array}$} & \multicolumn{4}{c}{ Pacientes } \\
\cline { 2 - 5 } & Fr $\%$ & \multicolumn{1}{c}{ Fra } & \%a \\
\hline \hline Mayor a 15 minutos & 16 & 20 & 16 & 20 \\
Menos de 15 minutos & 56 & 68 & 72 & 88 \\
No solicitaron & 10 & 12 & 82 & 100 \\
medicamentos & & & & \\
\hline \hline
\end{tabular}

Fuente: Elaboración propia. 
La satisfacción con el manejo del dolor en las mujeres con cesárea iterativa obtuvo una media de 11.3 con una desviación estándar de 0.9 , ligeramente mayor que las que tenían cesárea anterior y su primera cesárea. La satisfacción según la indicación médica fue mayor en las que tuvieron una cesárea electiva y según la técnica quirúrgica Beck y Kerr obtuvieron una media muy similar.

\section{Tabla 4}

Análisis Descriptivo del indicador Satisfacción con el manejo del Dolor

\begin{tabular}{l|c}
\hline \multicolumn{1}{c|}{$\begin{array}{c}\text { Análisis/ } \\
\text { Alternativas }\end{array}$} & Media $\pm \mathbf{D E}$ \\
\hline \hline & \\
Cesárea iterativa & $\mathbf{1 1 . 3} \pm \mathbf{0 . 9}$ \\
Cesaría anterior & $\mathbf{8 . 4} \pm \mathbf{1 . 2}$ \\
Primera cesárea & $\mathbf{9 . 4} \pm \mathbf{0 . 7}$ \\
& \\
\hline \hline
\end{tabular}

Fuente: Elaboración propia.

Además, fue posible develar que las mujeres que reportaron el dolor máximo en 24 horas fueron las que no recibieron orientación preoperatoria contra las que la recibieron ( 8.45 vs $7.03, p=.01)$, Se constató que las mujeres que estaban más satisfechas con el manejo del dolor, fueron las que recibieron orientación preoperatoria respecto a las que no las recibieron $(\mathrm{p}=.0)$. Asimismo, las mujeres que se les realizó la cesárea de urgencia presentaron mayor intensidad del dolor las primeras 24 horas, el dolor promedio y la interferencia entre la actividad general.

\section{Conclusiones}

La edad promedio de las pacientes en período de tiempo de recuperación completa del aparato reproductor después del parto fue de 23 años y el número de embarazos fue entre 2 y 3 .

El tipo de cesárea según el antecedente obstétrico que predominó fue la cesárea anterior y según la indicación fue la de urgencia y la técnica quirúrgica la Beck. 
Se evidencio que no existe un manejo adecuado del dolor en pacientes puerperio atendidas por el servicio de obstetricia, reflejado en la manifestación de dolor durante las primeras 24 horas al ser clasificado como severo y con un dolor promedio moderado.

Predomino el dolor moderado según su intensidad, mientras que el dolor máximo presentado en las primeras 24 horas fue clasificado como severo y dolor promedio fue clasificado como moderado.

La intensidad del dolor según las cirugías previas y las que recibieron orientación preoperatoria, fue clasificada por las mujeres del estudio como dolor moderado, en la que se destaca el hecho en la cual las mujeres con cirugía previa refirieron un dolor intenso, de forma similar lo reportan las mujeres que no recibieron orientación preoperatoria.

La satisfacción de la paciente con el manejo del dolor por parte del personal de salud, indicaron que recibieron mayor atención por parte del personal de enfermería.

El tiempo de espera para recibir el analgésico cuando reportaron las mujeres tener dolor, tardó menos de 15 minutos.

La satisfacción con el manejo del dolor en las mujeres con cesárea iterativa fue ligeramente mayor que las que tenían cesárea anterior y su primera cesárea. La satisfacción según la indicación médica fue mayor en las que tuvieron una cesárea electiva y según la técnica quirúrgica Beck y Kerr obtuvieron una media muy similar.

No hubo una relación significativa entre la satisfacción con el manejo del dolor por el personal de salud con la presencia de dolor durante la entrevista, indicando que no existe incidencia de asociación del dolor máximo en las 24 horas y el dolor promedio.

Las mujeres que reportaron el dolor máximo en 24 horas fueron las que no recibieron orientación preoperatoria, en la que constató que las mujeres que estaban más satisfechas con el manejo del dolor, fueron las que recibieron orientación preoperatoria respecto a las que no las recibieron.

La intensidad del dolor no se asoció con la satisfacción con el manejo del dolor. Las mujeres con cesáreas anteriores y que reportan que el personal de salud tardó menos de 10 minutos en atender su necesidad de dolor, refieren mayor satisfacción con el manejo del mismo. 


\section{Referencias Bibliográficas}

Covarrubias-Gómez A, Silva-Jiménez A, Nuche-Cabrera E, Téllez-Isaía

M (2006). El manejo del dolor postoperatorio en obstetricia: ¿Es seguro? Revista Mexicana de Anestesiología.

McCaffery M y Pasero C. (1999). Pain: Clinical Manual. St. Louis: Mosby;

Olapour A, Behaeen K, Akhondzadeh R (2013). The effect of inhalation of aromatherapy blend containing lavander essential oil on cesarean postoperative pain. Anesth Pain. 203-207

Paladino M A (2003). Accidentes, incidentes y complicaciones intraoperatorias. Rev Arg Anest.

Whizar Lugo V (2009). Prevención en Anestesiología. Anestesia en México. 